\title{
ÉDITORIAL
}

\section{Un an déjà ! Mais nous ne sommes qu'au premier étage}

\section{Heresbach}

(C) Springer-Verlag France 2010

Il y a un an, le conseil d'administration de la Société française d'endoscopie digestive (SFED) décidait, après le rachat par Springer de Acta endoscopica, de l'adopter comme revue officielle de la SFED. Son objectif a été de publier régulièrement les textes des conférences ou ateliers des réunions de la SFED ou des associations partenaires comme le Club français d'échoendoscopie digestive (CFED), le CREGG et la SMIER ou de réunions parrainées par la SFED. Cette orientation a pour but de lui garder sa vocation de revue de formation continue et d'information professionnelle proche de vos préoccupations quotidiennes, incluant la diffusion commentée de circulaires administratives relatives à l'exercice de l'endoscopie, le relais des modifications de l'EPP et les comptes rendus de congrès. Des rubriques comme les consensus en endoscopie digestive, les avis d'expert ou l'image du mois permettent d'encadrer notre exercice pour tendre vers un « service médical rendu » homogène, sous différents aspects, qu'ils soient formels (sans pour autant être opposables), anecdotiques ou classiques. Votre participation pour faire vivre Acta endoscopica est naturellement également sollicitée de façon classique par l'envoi d'articles originaux, de cas cliniques ou d'images du mois ; toutefois, si souvent on y pense et si parfois on en a l'envie, on en trouve rarement le temps, et on n'aboutit qu'exceptionnellement à la concrétisation.

C'est pourquoi nous vous proposons, cette année, de compléter ces rubriques par un « Endobuzz » afin que chacun puisse s'exprimer sous la forme d'un commentaire d'article court ou de résumé commenté sur les questions d'actualité que plusieurs parmi vous se posent. Nous espérons que cette nouvelle rubrique vous permettra de vous impliquer plus facilement dans notre revue. Il vous sera facile d'adresser vos écrits à l'un des trois fidèles et très actifs rédacteurs, C. Boustière, G. Lesur ou moi-même, dès votre choix. Afin d'éviter les doublons (oui, oui...), la règle sera de recevoir votre texte dans le mois suivant le dépôt du sujet ; au-delà de ce délai, le thème pourra être traité par un autre intéressé.

Une rubrique intitulée " Qu'auriez-vous fait » sera également mise en place et animée par un groupe multidisciplinaire, car l'endoscopie digestive est à un carrefour où convergent d'autres méthodes diagnostiques (en particulier radiologiques) et la cancérologie (aussi bien pour l'endoscopie diagnostique que thérapeutique).

L'année 2010 sera jalonnée de modifications professionnelles concernant le DPC, la circulaire 138 sur la désinfection et le stockage des endoscopes, la sédation, la gestion des antiagrégants, l'application de la check-list ; toutes ces modifications ne manqueront pas de susciter de nombreux commentaires, élans, frustrations ou débats. Le rôle d'Acta endoscopica, et à travers cette revue de la SFED, est de relayer le maximum d'opinions en gardant un cadre formel et une vérification des informations ou des commentaires publiés, au risque d'apparaître (trop) traditionnel. En effet, ne cédons pas à la mode de la peopolisation ou du sensationnel, dont les médias et leurs financeurs sont souvent friands pour attirer le client : les lecteurs sont nos partenaires, certes abonnés mais non-clients, et même si la publication ou l'édition nécessitent de réduire de plus en plus les moyens, gardons-nous d'alimenter la concurrence entre éditeurs ou pire entre sociétés savantes, associations ou groupes, comme certains maîtres d'actions caritatives très médiatisées s'y sont récemment hasardés.

L'été dernier, nous avons raté une marche de l'escalier : nos récents contacts avec des rédacteurs d'autres revues ou sociétés du savoir témoignent encore d'une certaine frilosité par peur de disparaître ou, au contraire, par désir de manger l'autre! Alors, ne nous prenons pas les pieds dans le tapis !

D. Heresbach $(\bowtie)$

Service des maladies de l'appareil digestif, CHU Pontchaillou,

2, rue Henri-Le-Guilloux, F-35033 Rennes cedex 09, France

e-mail : denis.heresbach@chu-rennes.fr 